\title{
Neurospora crassa developmental control mediated by the FLB-3 transcription factor
}

\author{
Ana Carolina Boni ${ }^{\mathrm{a}, 1}$, Daniela Luz Ambrósio ${ }^{\mathrm{a}, 1}$, Fernanda Barbosa Cupertino a \\ Alejandro Montenegro-Montero ${ }^{\mathrm{b}}$, Stela Virgilio ${ }^{\mathrm{a}}$, Fernanda Zanolli Freitas a, \\ Flávia Adolfo Corrocher ${ }^{a}$, Rodrigo Duarte Gonçalves a , Ally Yang c, \\ Matthew T. Weirauch d, e, Timothy R. Hughes c, f, g, Luis F. Larrondo b, \\ Maria Célia Bertolini ${ }^{\text {a, * }}$ \\ a Departamento de Bioquímica e Tecnologia Química, Instituto de Química, UNESP, Universidade Estadual Paulista, 14800-060, Araraquara, SP, Brazil \\ ${ }^{\mathrm{b}}$ Millennium Institute for Integrative Systems and Synthetic Biology (MIISSB), Departamento de Genética Molecular y Microbiología, Facultad de Ciencias \\ Biológicas, Pontificia Universidad Católica de Chile, Santiago 8331150, Chile \\ ${ }^{\mathrm{c}}$ Donnelly Centre, University of Toronto, Toronto, ON M5S 3E1, Canada \\ d Center for Autoimmune Genomics and Etiology (CAGE) and Divisions of Biomedical Informatics and Developmental Biology, Cincinnati Children's Hospital \\ Medical Center, Cincinnati, $\mathrm{OH} 45229$, USA \\ e Department of Pediatrics, University of Cincinnati College of Medicine, Cincinnati, OH 45229, USA \\ ${ }^{\mathrm{f}}$ Department of Molecular Genetics, University of Toronto, Toronto, ON M5S 1A8, Canada \\ g Canadian Institutes for Advanced Research, Toronto, ON, Canada
}

\section{A R T I C L E I N F O}

\section{Article history:}

Received 25 October 2017

Received in revised form

16 January 2018

Accepted 22 January 2018

Available online 1 March 2018

Corresponding Editor: Drauzio E.N. Rangel

\section{Keywords:}

Development control

FLB-3

Neurospora crassa

Protein-binding microarrays (PBM)

\begin{abstract}
A B S T R A C T
Here, we report that the Neurospora crassa FLB-3 protein, the ortholog of the Aspergillus nidulans FlbC transcription factor, is required for developmental control. Deletion of $f l b-3$ leads to changes in hyphae morphology and affects sexual and asexual development. We identified, as putative FLB- 3 targets, the $N$. crassa aba-1, wet-1 and vos-1 genes, orthologs of the ones involved in A. nidulans asexual development and that work downstream of FlbC ( $a b a A$, wetA and vosA). In $N$. crassa, these three genes require FLB-3 for proper expression; however, they appear not to be required for normal development, as demonstrated by gene expression analyses during vegetative growth and asexual development. Moreover, mutant strains in the three genes conidiate well and produce viable conidia. We also determined FLB-3 DNA-binding preferences via protein-binding microarrays (PBMs) and demonstrated by chromatin immunoprecipitation (ChIP) that FLB-3 binds the aba-1, wet-1 and vos-1 promoters. Our data support an important role for FLB-3 in N. crassa development and highlight differences between the regulatory pathways controlled by this transcription factor in different fungal species.
\end{abstract}

(๔) 2018 British Mycological Society. Published by Elsevier Ltd. All rights reserved.

\section{Introduction}

The FlbC transcription factor was first described in Aspergillus nidulans, together with five other developmental activators of asexual spore formation (FluG, FlbA, FlbB, FlbD and FlbE) (Wieser et al., 1994; Adams et al., 1998). Mutation in any of these genes results in undifferentiated masses of vegetative hyphae that lead to the formation of cotton-like colonies described as "fluffy". FlbC has two $\mathrm{C}$-terminal $\mathrm{C}_{2} \mathrm{H}_{2}$ zinc finger DNA binding-domains and, in

\footnotetext{
* Corresponding author. Fax: +55 1633222308.

E-mail address: mcbertol@iq.unesp.br (M.C. Bertolini).

1 Both authors contributed equally to this work.
}

A. nidulans, is described to regulate development by activating the first gene of the conidiation-specific regulatory pathway, brlA, and by inhibiting vegetative growth (Kwon et al., 2010; Park and Yu, 2012). The brlA gene encodes a transcription factor that is necessary for development (Adams et al., 1988), activating a cascade of genes that characterize the so-called central regulatory pathway, required for conidiation (Park and Yu, 2012). In A. nidulans, the central regulators of conidiation include the $a b a A$ gene, a transcription factor necessary for normal conidiophore development and conidia formation (Adrianopoulos and Timberlake, 1994) and wet $A$, which is necessary for the synthesis of cell wall layers required for conidia maturation and impermeabilization (Marshall and Timberlake, 1991). The VosA and VelB regulators have more 
recently been shown to be connected to the central regulatory genes, acting as negative regulators of conidiation by exerting negative regulation over brlA (Park and Yu, 2012; Ni and Yu, 2007; Park et al., 2012). In addition to its aforementioned role in development, VosA has also been described to control trehalose biogenesis, thus coupling trehalose biosynthesis and conidia maturation (Ni and Yu, 2007).

FlbC orthologous have been characterized in only a few fungi. The fle1 gene encodes the FlbC ortholog in Podospora anserina (FLE1), which acts as a repressor of female sexual differentiation (Coppin, 2002). More recently, FlbC orthologs have been characterized in Fusarium verticillioides (Ada1) and in Penicillium oxalicum (PoFlbC). The $\Delta a d a-1$ mutant strain displays reduced production of conidia and defects in conidia and conidiophore morphology (Malapi-Wight et al., 2014). In P. oxalicum, PoFlbC is required for normal growth and asexual development, and gene deletion results in reduced production of cellulases, hemicellulases, and proteins related to lignocellulose degradation (Yao et al., 2016). In Neurospora crassa, the $A$. nidulans FlbC orthologous protein [formerly known as acon-4 (Chung, 2011) and herein termed flb-3], encoded by the ORF NCU03043, was identified as a putative transcription factor involved in the control of glycogen metabolism. The $\Delta f l b-3$ strain exhibits higher levels of glycogen and higher expression of the gene encoding glycogen synthase than the wild-type strain under heat stress (Gonçalves et al., 2011).

Although the FLB-3 transcription factor has been described as required for proper regulation of glycogen metabolism (Gonçalves et al., 2011), no reports have evaluated whether it plays any role in development in $N$. crassa. Conidiation in $N$. crassa involves a sequence of events that leads to the production of multinucleated macroconidia, uninucleated microconidia and arthroconidia (Springer and Yanofsky, 1989). Under specific environmental conditions, aerial hyphae grow and branch to form an aerial mass, from which conidiophores develop (Springer and Yanofsky, 1989). Many genes are activated in this process, each one participating at a specific stage. Light promotes this process through the activation of conidiation-specific genes, while the heterotrimeric $G$ protein signaling pathway also plays a role in the regulation of conidiation (Kays and Borkovich, 2004; Park and Yu, 2012). In a screen for genes differently expressed during asexual development, a large number of genes were identified (Greenwald et al., 2010), within which there were many encoding transcription factors, including $f l b-3$ (NCU03043), suggesting that they may play a role in conidiation.

In this work, we demonstrate that FLB-3 is a sequence-specific DNA-binding protein required for proper vegetative growth and sexual and asexual development in $N$. crassa. We identify the orthologs of $A$. nidulans developmental regulators $a b a A$ and wetA (termed $a b a-1$ and wet-1, respectively), but not the brlA ortholog. We further show that both genes, together with vos-1, are regulated by FLB-3 during vegetative growth and asexual development in $N$. crassa. In addition, we provide evidences for cross regulation between $a b a-1$, wet- 1 and vos-1 genes, which differs from the one described previously in A. nidulans. Interestingly, and contrary to that reported in $A$. nidulans, these genes appear not to be required for development in $N$. crassa. This work supports an important role for FLB-3 in $N$. crassa development and highlights differences between the regulatory pathways of conserved processes among different fungal species.

\section{Materials and methods}

\subsection{N. crassa strains and growth conditions}

The $N$. crassa wild-type (FGSC\#2489), $\Delta$ flb-3 (FGSC\#11355, NCU03043), $\Delta$ vos-1 (FGSC\#13536, NCU05964), swet-1 (FGSC\#11204,
NCU01033), and $\Delta a b a-1$ (FGSC\#15901, NCU02612) strains were purchased from the Fungal Genetics Stock Center (FGSC) (McCluskey, 2003). All strains were maintained on solid Vogel's minimal (VM) medium (Vogel, 1956), pH 5.8 containing $2 \%$ sucrose. To induce protoperithecia formation, the wild-type and $\Delta f l b-3$ strains were grown on $0.1 \times$ SC medium (Westergaard and Mitchell, 1947) containing $0.5 \%$ sucrose. For microconidiation assay, the same medium was used, but supplemented with $1 \mathrm{mM}$ sodium iodoacetate (Ebbole and Sachs, 1990). Conidia from 10-d cultures were collected, suspended in sterile water and counted in a Neubauer chamber. Microconidia were isolated from 10 to 15 -d cultures after filtration using Millex SV (5 $\mu \mathrm{m})$ filters.

To induce asexual development, $10^{6}$ conidia $\mathrm{ml}^{-1}$ from the wildtype strain or a conidia suspension (from the $\Delta f l b-3$ strain) were inoculated into liquid VM medium $(125 \mathrm{ml})$ plus $2 \%$ sucrose and grown at $30^{\circ} \mathrm{C}$ and $200 \mathrm{rpm}$ for $20 \mathrm{~h}$ (control, time $0 \mathrm{~h}$ ). After this, mycelia were filtered in order to have a thin layer. Mycelial discs were cut and placed onto the surface of a petri dish containing solid VM medium plus $2 \%$ sucrose, over a cellophane membrane, and exposed to constant light at $25^{\circ} \mathrm{C}$ for different times $(6,9,12$, and $24 \mathrm{~h}$ ) in a Percival Scientific incubator. All samples were frozen in liquid nitrogen and stored at $-80^{\circ} \mathrm{C}$ for RNA extraction.

For microscopy analysis, $10^{7}$ microconidia $\mathrm{ml}^{-1}$ from wild-type and a conidial suspension from the $\Delta f l b-3$ strain were germinated for different times within plates containing coverslips immersed in liquid VM medium plus $2 \%$ sucrose. For morphological hyphae analysis, mycelia were stained with $100 \mu \mathrm{L}$ of calcofluor white (3.7 $\mu \mathrm{g} \mathrm{ml}^{-1}$ final concentration) for $15 \mathrm{~min}$ and washed twice with phosphate-buffered saline (PBS). Fluorescence was visualized using a fluorescence microscope with excitation and emission wavelengths of $359 \mathrm{~nm}$ and $461 \mathrm{~nm}$, respectively. For nuclei analysis, mycelia were fixed [3.7\% formaldehyde, $50 \mathrm{mM} \mathrm{NaH}_{2} \mathrm{PO}_{4}, \mathrm{pH} 7.0$, $0.2 \%(\mathrm{v} / \mathrm{v})$ tween 80 ], washed twice with PBS, stained with $100 \mu \mathrm{L}$ DAPI $\left(0.5 \mathrm{mg} \mathrm{ml}^{-1}\right)$ for $5 \mathrm{~min}$ and visualized as described above. In both cases, hyphae were examined in an AXIO Imager.A2 Zeiss microscope. All images were captured with the AxioCam MRm camera and processed using the AxioVision software, version 4.8.2. For apical hyphae analysis, $10^{7}$ conidia $\mathrm{ml}^{-1}$ were inoculated onto plates containing solid VM medium for $20 \mathrm{~h}$. Images of colony morphology were captured with an AxioCam ICc3 camera coupled to the stereoscope trinocular Discovery V8 (Zeiss) at $50 \times$ magnification.

For vegetative growth experiments, $10^{7}$ conidia $\mathrm{ml}^{-1}$ from wildtype strain or hyphae and conidia homogenates from the $\Delta f b-3$ strain were inoculated into VM medium ( $125 \mathrm{ml}$ ) plus $2 \%$ sucrose at $30{ }^{\circ} \mathrm{C}, 200 \mathrm{rpm}$, and mycelia pads were collected every $12 \mathrm{~h}$. To count microconidia, $25 \mu$ l of suspension with $10^{7}$ conidia from wildtype, the FLB-3 overexpressing strain and the mutant strain were inoculated onto Erlenmeyer flasks containing solid VM medium plus $2 \%$ agar and $2 \%$ sucrose at $\mathrm{pH} 5.8$ for $9 \mathrm{~d}$. Conidia were collected and suspended in $30 \mathrm{ml}$ of sterile water, and $250 \mu \mathrm{l}$ from this conidia suspension were filtrated using Millex SV $(5 \mu \mathrm{m})$ filters and counted. Experiments were repeated four times.

\section{2. $R N A$ isolation and gene expression analysis}

Total RNA was prepared from mycelia samples (Sokolovsky et al., 1990). RNA ( $1 \mu \mathrm{g})$ from each sample was fractionated onto a $2.2 \mathrm{M}$ formaldehyde $1.2 \%(\mathrm{w} / \mathrm{v}$ ) agarose gel, stained with ethidium bromide, and visualized under UV light to assess rRNA integrity. Gene expression analysis was performed by quantitative PCR (RT-qPCR). RNA samples $(10 \mu \mathrm{g})$ were treated with RQ1 RNAse-free DNAse (Promega) and subjected to cDNA synthesis using the SuperScript III First Strand Synthesis kit (Invitrogen) and oligo (dT) according to the manufacturer's instructions. RT-qPCR was performed on a StepOnePlus тмReal-Time PCR System (Applied Biosystems) using 
the Power SYBR ${ }^{\circledR}$ Green PCR Master Mix (Applied Biosystems) and specific primers for $f l b-3, a b a-1$, vos-1, and wet-1 mRNA amplicons (see Supplementary Table S1 online, qPCR). Data analysis was performed using the Comparative $C T(\Delta \Delta C T)$ method as implemented in StepOne Software (Applied Biosystems) (Livak and Schmittgen, 2001). Data were normalized to the reference gene $\beta$-tubulin (tub-2, NCU04054). All reaction efficiencies were between 94 and $100 \%$. For each sample, at least three biological replicates were performed.

\subsection{Determination of the sequence preferences of $F L B-3$ via protein binding microarrays and identification of putative FLB-3 target sites}

We used protein-binding microarrays (PBM) (Berger et al., 2006) to determine the sequence preferences of the FLB-3 protein. We designed primers to clone, via conventional PCR methods, a region encompassing the predicted $\mathrm{C}_{2} \mathrm{H}_{2}$ DNA-binding domain of FLB-3 (as identified using HMMER; Eddy, 2009), from residues 313 to 385 , into the T7-GST vector for expression in Escherichia coli (Weirauch et al., 2014). The construct was analyzed in duplicate on two different arrays with differing probe sequences (denoted "ME" and "HK"). Calculation of 8-mer $Z$ and $E$ scores, which estimate the strength of binding to each possible 8-mer, was performed as previously described (Berger et al., 2006). The FLB-3 binding motif was derived using the BEEML-PBM method (Zhao and Stormo, 2011; Weirauch et al., 2013).

We analyzed the $3.5 \mathrm{~kb}$ upstream region of the genes of interest (3.5 kb upstream of the predicted translational start site, Neurospora genome annotation NC12) for the identification of putative FLB-3 binding sites. Sequences were scored with the PBMdetermined PWM using the standard log-likelihood scoring scheme, with a cutoff of 7 bits (Stormo, 1990). Motif comparisons were performed with Tomtom (Gupta et al., 2007), version 4.10.1 (as implemented at http://meme-suite.org/tools/tomtom) using the Sandelin-Wasserman similarity function with default settings (Wasserman and Sandelin, 2004).

\subsection{Construction of the FLB-3 overexpressing strain}

To generate the sfGFP-tagged FLB-3 strain, a DNA fragment of 1269 bp was amplified by PCR with the primers C-sfGFPFLBC-F and C-sfGFPFLBC-R (Table S1) using genomic DNA from the wild-type strain as template. The C-sfGFPFLBC-R oligonucleotide contains the sequence that encodes for 6-Gly that links the FLB-3 coding sequence and sfGFP. PCR was performed using Phusion High-Fidelity PCR kit (Finzymes) and the DNA fragment was purified with QIAquick Gel Extraction Kit (QIAGEN, CA), according to the manufacturer's instructions. The purified DNA fragment was cloned into the Xbal and Pacl sites of the pTSL91-A plasmid (a donation from N. L. Glass, University of California at Berkeley, Berkeley, CA, USA), leading to the pTSL91-A-flb-3 construct. The pTSL91-A plasmid allows for constitutive expression of the C-terminus tagged FLB-3-sfGFP fusion protein under the control of the $c c g-1$ promoter. The pTSL91-A-flb-3 construct was used to transform competent conidia from a recipient his-3 mutant strain (FGSC\#9716, mat a his $-3^{-}$). The transformants (his-3::Pccg-1-flb-3-sfgfp) were selected on VM media without histidine. The homokaryons were isolated after crossing with the wildtype strain (FGCS\#2498, mat A), and confirmed by PCR using the primers C-sfGFPFLBC-F and C-sfGFPFLBC-R (Table S1). The his3::Pccg-1-flb-3-sfgfp was named $f l b-3^{+}$

\subsection{Chromatin immunoprecipitation-PCR assays}

Chromatin immunoprecipitation (ChIP) assays were performed as previously described (Tamaru et al., 2003). Briefly, $10^{8}$ conidia from the $f l b-3^{+}$strain were grown in different flasks containing $125 \mathrm{ml}$ of VM liquid medium with $2 \%$ sucrose, $\mathrm{pH} 5.8$, and incubated at $30{ }^{\circ} \mathrm{C}$ and $200 \mathrm{rpm}$ during 12, 24, 48 and $60 \mathrm{~h}$. Samples were fixed by adding formaldehyde (Sigma) to a final concentration of $1 \%$, and the formaldehyde was quenched with glycine. Samples were suspended in ChIP lysis buffer and the procedure followed the conditions previously described (Freitas et al., 2016). The DNA was quantified using Nanovue Plus (GE Healthcare) and 200 ng of Input DNA (IN), no Ab (N, reaction without antibody) and IP [immunoprecipitated DNAs with anti-GFP antibody (Sigma)] samples were amplified by PCR using primers specific for each promoter (Table S1, ChIP-PCR). Input DNA was used as a positive control and no Ab as negative control of the experiments.

PCR was performed using Phusion High-Fidelity PCR kit (Finzymes) and specific oligonucleotides (see Supplementary Table S1 online, ChIP-PCR) for paba-1 (pabaFLBC-F/pabaAFLBC-R), pvos-1 (pVOS1FLBC-F/pVOS1FLBC-R) and pwet-1 (pWETAFLBC-F/pWETAFLBC-R) promoters. A region containing a match of the A. nidulans FlbC motif in the vos-1 promoter was analyzed using the primers pvosA (pVosA-F/pVosA-R). Reactions were performed under the following conditions: $98^{\circ} \mathrm{C}$ for $10 \mathrm{~s}, 35$ cycles of $98^{\circ} \mathrm{C}$ for $1 \mathrm{~s}$, $60{ }^{\circ} \mathrm{C}$ for $5 \mathrm{~s}$ and $72{ }^{\circ} \mathrm{C}$ for $30 \mathrm{~s}$, and then $72{ }^{\circ} \mathrm{C}$ for $5 \mathrm{~min}$. The reaction products were analyzed on a $2 \%$ agarose gel and visualized with ethidium bromide. Densitometry was performed using Image] software, and the IP signals were compared to the negative control $(\mathrm{N}$, no $\mathrm{Ab})$.

\section{Results}

\subsection{The ORF NCU03043 encodes the N. crassa ortholog of the A. nidulans FlbC transcription factor}

The NCU03043 gene is annotated as " $\mathrm{C}_{2} \mathrm{H}_{2}$ finger domaincontaining protein FlbC" in the $N$. crassa genome database (http:// fungidb.org/fungidb/app/record/gene/NCU03043). The predicted protein is composed of 385 amino acid residues and contains two typical $\mathrm{C}_{2} \mathrm{H}_{2}$ domains separated by a linker, which conforms to the canonical TGE[K/R]P[F/Y]X pattern. This protein exhibits substantial sequence similarity to functionally characterized FlbC orthologs from different fungi (Fig. S1), particularly in the predicted DNA-binding domain, and is syntenic with FlbC (http://fungidb.org/fungidb/app/ record/gene/NCU03043), suggesting that NCU03043 encodes the $N$. crassa ortholog of the A. nidulans FlbC transcription factor.

\subsection{Deletion of flb-3 results in morphological defects and impairment in sexual and asexual development}

To characterize the putative $N$. crassa ortholog of the A. nidulans FlbC transcription factor, we analyzed the morphological aspects of the $N$. crassa $\Delta$ flb-3 (NCU03043) strain. In A. nidulans, FlbC deletion results in a fluffy phenotype (cotton-like colonies) and defects in vegetative growth and asexual development, with reduced conidiation (Kwon et al., 2010; Park and Yu, 2012). Similarly, deletion of $f l b-3$ in $N$. crassa results in multiple morphologic defects related to growth and development, including low production of macroconidia and an inability to develop normal aerial hyphae, which lead to the generation of masses of vegetative hyphae with the same fluffy phenotype described for the A. nidulans mutant strain. In addition, the $\Delta f l b-3$ strain appears to have an apparently reduced $\beta$-carotene pigmentation (Fig. 1A). Moreover, analysis of apical hyphae in solid VM medium showed a high quantity of short hyphae, most likely resulting from hyperbranching (Fig 1B, top), and the very few macroconidia produced by the mutant strain displayed abnormal morphology (Fig. 1B, middle). We also evaluated whether FLB-3 impacts sexual development. Induction of female 


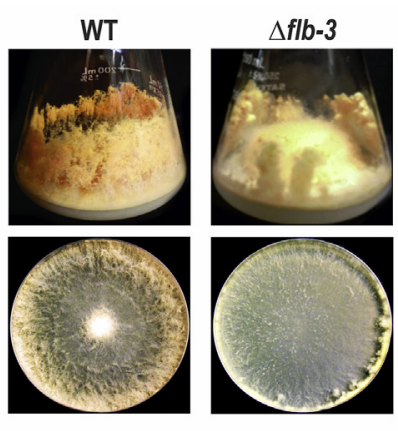

B
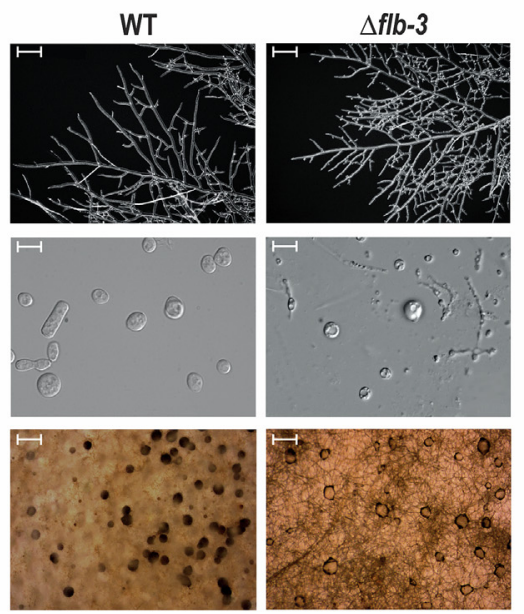

C

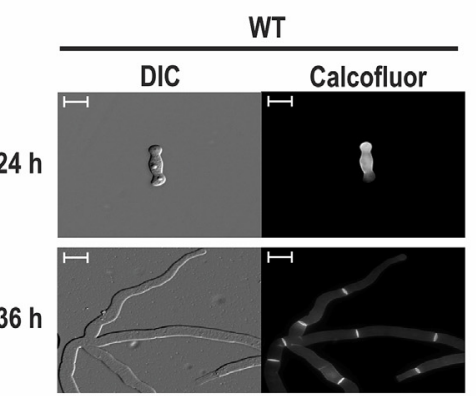

$48 \mathrm{~h}$

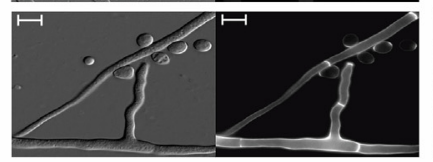

$\Delta f l b-3$
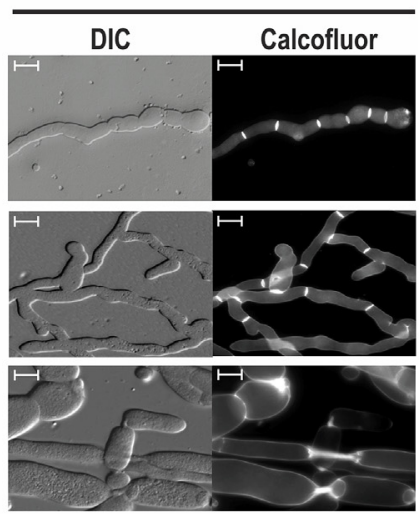

D

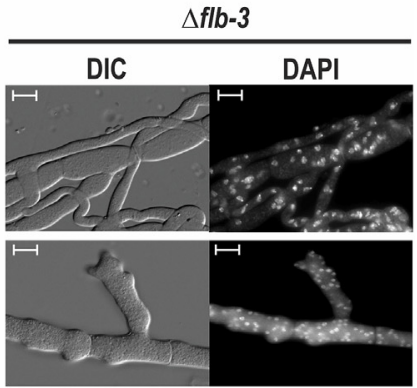

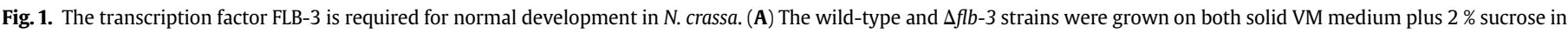

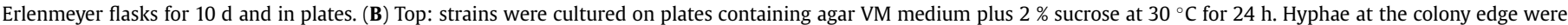

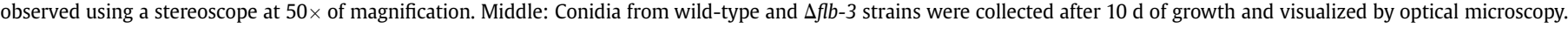

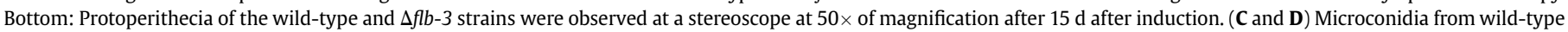

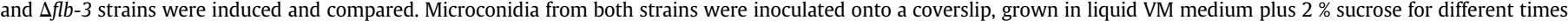
and stained with either calcofluor $(\mathbf{C})$ or DAPI (D). Images were obtained using the microscope AXIO Imager.A2 (Zeiss) with $100 \times$ magnification. Scale bar, $10 \mu$ m.

sexual development by nitrogen starvation, which normally results in highly pigmented protoperithecia, led to the production of an increased number of translucent protoperithecia in the $\Delta f l b-3$ strain compared to the wild-type strain (Fig. 1B, bottom). These were unable to engage in a cross with conidia from the opposite mating type, suggesting female sterility. We further tested the ability of the mutant strain to participate in a cross by using it as the male strain, but no crossing was observed, suggesting that deletion of $f l b-3$ results in complete sterility.

Conidial germination of the $\Delta f l b-3$ strain was also evaluated using microconidia from wild-type and mutant strains. Since the mutant strain produces a few conidia, microconidiation was induced in both strains and the cells were compared by microscopic analysis. Germination of wild-type and $\Delta f l b-3$ microconidia was compared by growing the cells in liquid VM medium for different time periods. Calcofluor White staining revealed that the $\Delta f l b-3$ microconidia germinated earlier than wild-type microconidia, however, the hyphae were shorter and displayed increased septation (Fig. 1C). After $48 \mathrm{~h}$ of germination, the $\Delta f l b-3$ strain exhibited severe morphological alterations, such as cytoplasmic constrictions and hyphae swelling (Fig. 1C and D).

\subsection{Expression of flb-3 is regulated during vegetative growth and asexual development}

As FLB-3 was demonstrated to be involved in vegetative growth and asexual development, we decided to analyze its expression under such growth conditions. In N. crassa, growth on liquid medium, under agitation, suppresses conidiation, enabling the evaluation of gene expression specifically under vegetative growth. The $f l b-3$ transcript started to accumulate at an early phase of vegetative growth, being highly expressed at later times and reaching highest levels at the latest time we assessed (60 h, Fig. 2A). To evaluate gene expression during asexual development, we transferred mycelia grown for $20 \mathrm{~h}$ on liquid cultures (C, control) to solid media and then exposed the cultures to light, which are conditions that induce conidiation in $N$. crassa (Greenwald et al., 2010). Under this growth condition, flb-3 expression decreased with time (Fig. 2B). Altogether, these results suggest that the FLB-3 transcription factor may play a function in vegetative growth and asexual and sexual development in $N$. crassa.

\subsection{Determination of the DNA-binding specificity of FLB-3}

To further characterize this transcription factor, we determined its DNA-binding preferences using Protein Binding Microarrays (PBMs), a robust and powerful system that allows for rapid and unbiased characterization of the sequence specificity of DNAbinding proteins (Andrilenas et al., 2015; Berger et al., 2006; Weirauch et al., 2014). Briefly, PBMs are microarrays containing $>40000$ double-stranded DNA probes, each 60 nucleotides in length. The probes are designed such that every possible nonpalindromic 8-base sequence appears on the array in 32 different flanking sequence contexts (palindromes occur 16 times). A GST- 

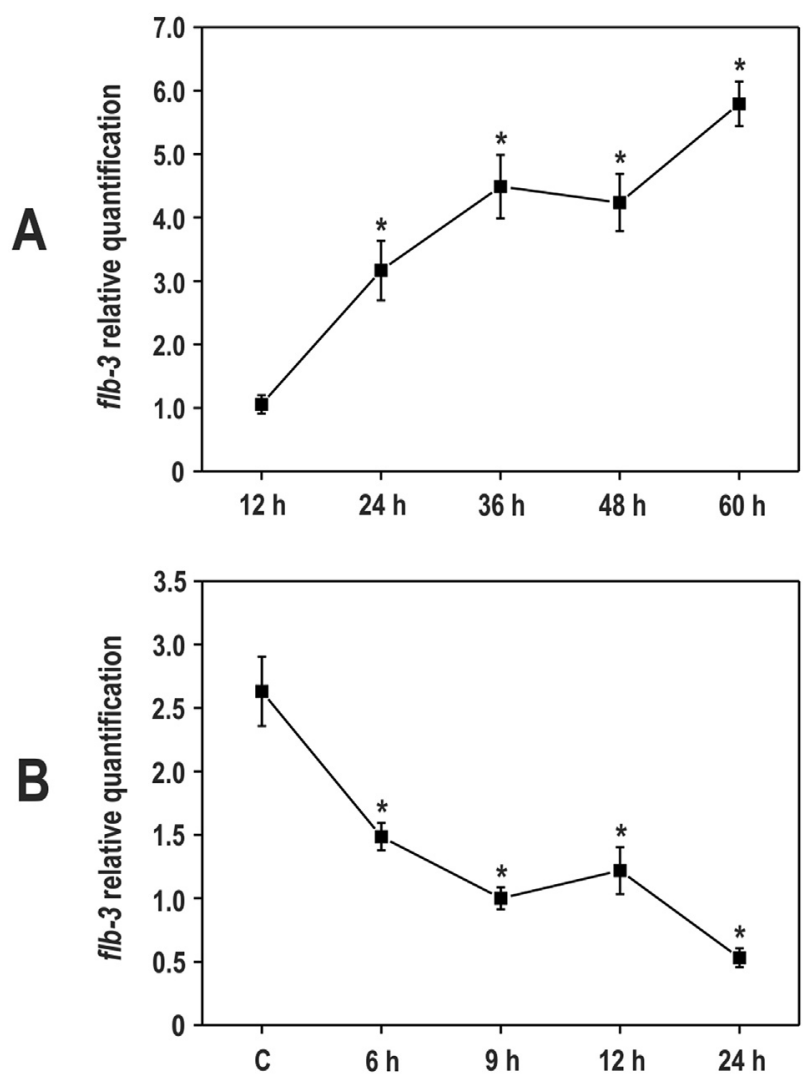

Fig. 2. Expression of the $f l b-3$ gene is developmentally regulated. (A and B) Gene expression analysis of $f l b-3$ in the wild-type strain during $(\mathbf{A})$ vegetative growth and (B) asexual development. For vegetative growth, conidia were inoculated into liquid VM medium and mycelia samples were harvested at different times. For asexual development, conidia were inoculated into liquid VM medium, collected by filtration and placed onto solid VM medium over a cellophane membrane and exposed to light for different times. Total RNA was extracted and gene expression analyzed by RT-qPCR The tub-2 gene (NCU04054) was used as the reference gene in all experiments. Data shown represent the means and standard deviations of the results of three independent experiments. $\left(^{*}\right)$ Asterisks indicate the statistically significant differences according to Tukey-Kramer multiple comparison test $(p<0.001)$ between the levels at each time point compared to those after $12 \mathrm{~h}(\mathbf{A})$ and to the one in $\mathrm{C}(\mathbf{B})$ tagged protein consisting of the predicted FLB-3 DNA binding domain and flanking sequence is then bound to the array, the amount of binding to each probe is quantified based upon the measured GST fluorescence, and used to construct DNA binding models (see Methods). Through this approach, we determined that FLB-3 is indeed a sequence-specific DNA-binding protein and characterized its binding specificity (Fig. 3A).

The obtained FLB-3 motif is only somewhat similar to the one reported for FlbC (TGACGAT/A) (Kwon et al., 2010), the A. nidulans ortholog of FLB-3. This is surprising, considering the high identity exhibited by these two proteins in their DNA-binding domains (93.8\% of their amino acids are identical in this region), which usually results in similarities in their DNA sequence preferences (Weirauch et al., 2014; Bernard et al., 2012). This led us focus on the reported FlbC motif further. The published FlbC motif was derived from the inspection of only three promoter regions, and the motif discovery was limited to searching for sequence matches similar to the characterized DNA-binding preferences of the Saccharomyces cerevisiae CMR3/YPR013C (Zhu et al., 2009) (available at http:// cisbp.ccbr.utoronto.ca), a putative $\mathrm{C}_{2} \mathrm{H}_{2}$ zinc finger protein, which exhibits certain similarity to FlbC within the zinc finger domain (Kwon et al., 2010). The reported FlbC motif however, displays only a minor overall similarity to the one derived for the yeast protein (Tomtom p-value 0.25). The herein determined FLB-3 motif, on the other hand, displays a significantly more similar motif to that determined for YPR013C (Tomtom p-value 3.8e-05), consistent with their similarity in the predicted DNA-binding region, suggesting that the binding specificity we obtained from the PBM may more accurately represent the specificity of FlbC-like proteins.

Several groups have developed methods to predict the DNAbinding specificity of zinc finger proteins based on the specific residues present at canonical positions within the ZF domains. As an additional test to evaluate and compare our motif to that reported for FlbC, we used a recently published random forest-based predictive recognition model for zinc finger proteins, ZFModels (Gupta et al., 2014), which takes the full sequence of a protein of interest, and through an HMM-based algorithm, extracts the determinants in each finger for construction of a recognition motif. The ZFModels-derived motif predicted for either FLB-3 or FlbC is identical (again, consistent with their similarity in the DNA-binding region) and is largely in agreement with our PBM-derived motif for

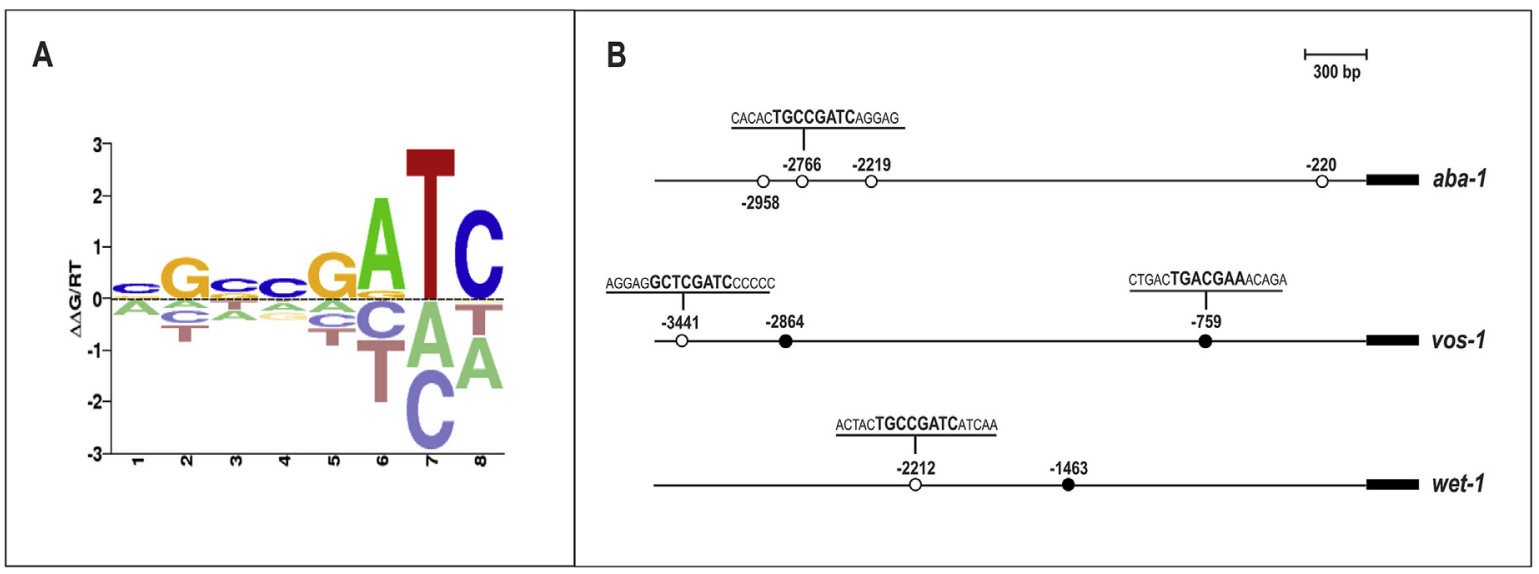

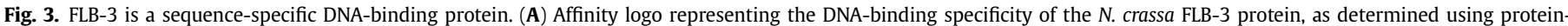

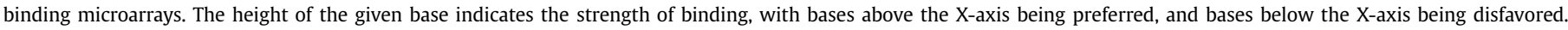

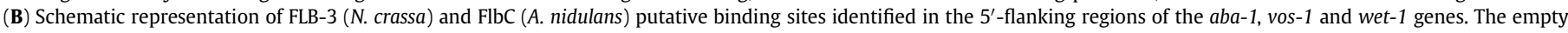

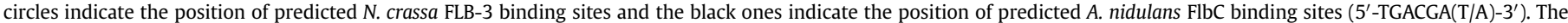

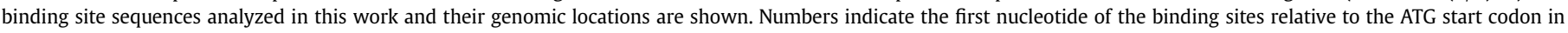
the + strand. 
FLB-3 (Tomtom p-value 0.0016). The similarity with the published FlbC motif, however, is low (TomTom p-value 0.58). As expected, the ZFModels motif for FLB-3 is also significantly similar to the PBM-determined one for YPR013C (Tomtom p-value 0.0053). These results largely agree with those obtained with another predictive method, B1H-RC (Najafabadi et al., 2015) (not shown). Altogether, this suggests that the sequence preference of FlbC and FlbC-like proteins may be somewhat different from what has been reported and may actually resemble more closely the one we determined for FLB-3, which would be consistent with their similarity in the DNA-binding region (Weirauch et al., 2014; Bernard et al., 2012), and general predictive models of the sequence specificity of zinc finger proteins.

\subsection{Identification of the A. nidulans AbaA, WetA and VosA orthologs in N. crassa}

In A. nidulans, it has been proposed that FlbC activates brlA, which encodes for the early regulator of development, BrlA, which then activates $a b a A$ and wetA. These three genes coordinately regulate the expression of a variety of conidiation-specific genes (Adams et al., 1988). In addition, the regulators VosA and VelB are interconnected to these central regulatory genes (Park and Yu, 2012). The VosA regulator has additionally been described to control trehalose biogenesis ( $\mathrm{Ni}$ and $\mathrm{Yu}, 2007$ ). To further characterize the pathway involving FLB-3, we searched for the $N$. crassa genes that are predicted to be downstream of FLB-3, based on the A. nidulans conidiation pathway. A BLASTP search in the $N$. crassa database identified putative orthologs for some of these genes (termed here as aba-1, wet-1 and vos-1). We were unable however, to identify a putative brlA ortholog or the putative orthologs of other known A. nidulans developmental regulators (sfgA and velB) (Fig. 4A).

The NCU02612 predicted protein was identified as the A. nidulans AbaA ortholog (41\% identity, termed here as ABA-1). This protein is characterized as a DNA-binding protein belonging to the TEA/ATTS domain family of transcriptional regulators (Adrianopoulos and Timberlake, 1991). This domain has been identified and characterized in a few regulatory proteins (Laloux et al., 1990; Xiao et al., 1991; Campbell et al., 1992). Sequence alignment of ABA-1 and orthologous proteins from different filamentous fungi is shown in Fig. S2. ABA-1, together with the Sordaria macrospora ABAA protein, are the largest among the analyzed proteins and share identical amino acids residues in the TEA/ATTS domain (Adrianopoulos and Timberlake, 1991). The NCU01033 predicted protein, annotated as a hypothetical protein, was identified as an ortholog of the $A$. nidulans WetA protein (65\% identity, termed here as WET-1), and Fig. S3 shows the sequence alignment of related proteins from different filamentous fungi. The NCU05964 protein is already annotated in the $N$. crassa database as the developmental regulator VOS-1, the putative VosA ortholog. This protein contains a velvet domain in the $\mathrm{N}$-terminal region, which corresponds to a new class of DNA-binding domain present in transcription factors classified under the velvet family ( $\mathrm{Ni}$ and $\mathrm{Yu}$, 2007). The velvet regulators are highly conserved proteins, only found in ascomycetes and basidiomycetes fungi (Bayram and Braus, 2012). They have been described to play a role in various processes, such as developmental control, sporogenesis and secondary metabolism (Park et al., 2012; Bayram and Braus, 2012).

To evaluate the role of these genes in N. crassa, the morphology of the $\Delta a b a-1, \Delta v o s-1$ and $\Delta$ wet- 1 strains was compared to that of the wild-type strain. Despite some slight differences among them

A

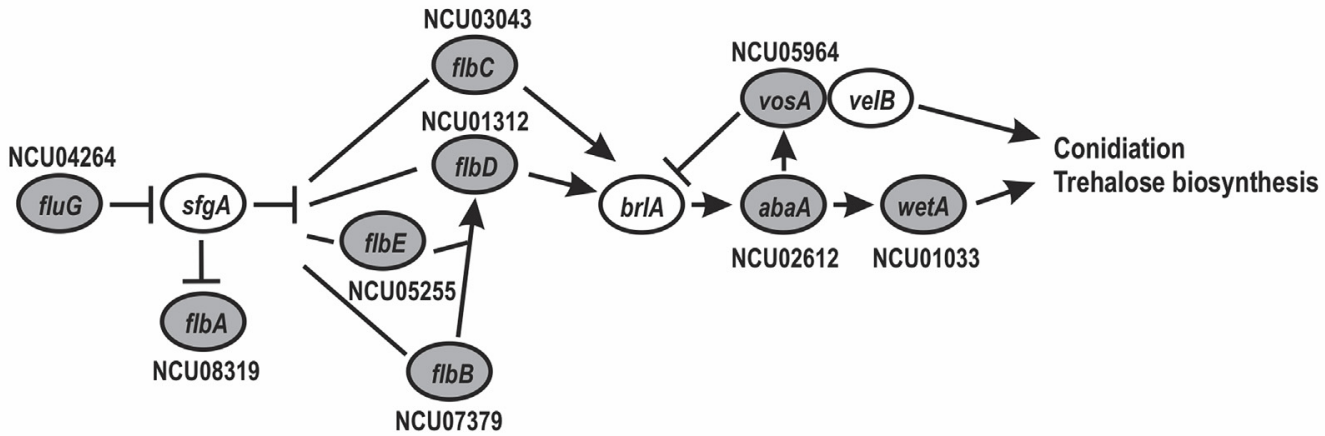

B
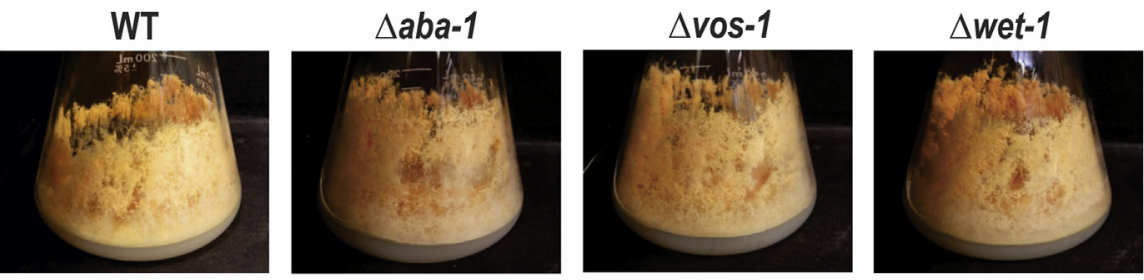

9-days culture
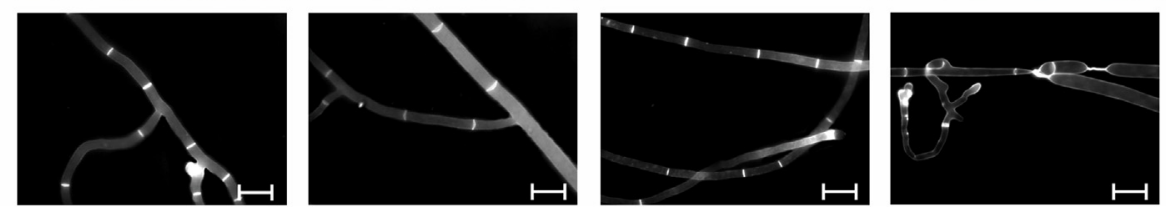

$12 \mathrm{~h}$ after germination

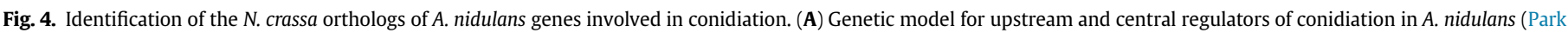

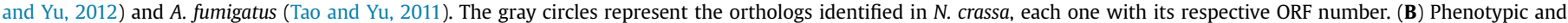

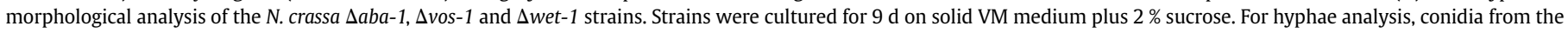
strains were germinated in liquid VM medium plus $2 \%$ sucrose for $12 \mathrm{~h}$ and stained with calcofluor white. 
(e.g. rare hyphae constrictions in the $\Delta$ wet-1 strain), they were phenotypically similar to the wild-type strain after growth on minimal solid medium and conidial germination (Fig. 4B). All strains exhibited viable conidia and the conidia germinated well, with the exception of $\Delta v o s-1$, which displayed a slight delay in conidia germination, but was able to reach normal hyphae growth after $8 \mathrm{~h}$ of incubation in liquid culture (data not shown). We further analyzed conidia viability after storage in all strains and they retained high viability (around $90 \%$ ) after up to $21 \mathrm{~d}$ at $4{ }^{\circ} \mathrm{C}$ (data not shown). The lack of vegetative growth phenotypes in the aforementioned mutants was unexpected, based on what is known about the corresponding orthologs in Aspergilli (Adrianopoulos and Timberlake, 1994; Tao and Yu, 2011; Son et al., 2013), and suggests divergent functionality between the $N$. crassa proteins and those from others filamentous fungi regarding the central regulatory pathway of conidiation: $a b a-1$, wet- 1 and vos- 1 may not play such important roles in $N$. crassa conidiation as they do in Aspergilli. The result related to $a b a-1$ gene here is consistent with the one reported by Chung (2011). The $N$. crassa gene does not play an analogous role in conidiation as the $A$. nidulans gene; it does not complement defective conidiation in A. nidulans abaA14 mutant, even using different promoters.

\subsection{FLB-3 regulates aba-1, wet- 1 and vos- 1 expression during vegetative growth and asexual development and binds to their promoters}

In A. nidulans, FlbC regulates the expression of genes of the conidiation pathway (Kwon et al., 2010). To verify whether FLB-3 is required for the normal expression of $a b a-1$, wet- 1 and vos-1, we analyzed the expression of all these genes in the wild-type and $\Delta f l b-3$ strains by RT-qPCR. The assays were performed during vegetative growth in liquid submerged cultures and under induction of asexual development. During vegetative growth, the $N$. crassa wild-type strain displayed a maximum level of $a b a-1$ mRNA at $12 \mathrm{~h}$, and a reduction after this time, whereas the expression of vos-1 and wet-1 reached their highest levels after $48 \mathrm{~h}$ of growth (Fig. 5A). In the $\Delta$ flb-3 strain, the levels of the $a b a-1$ and wet- 1 transcripts were slightly lower than those in the wild-type strain and remained almost constant over the time period analyzed, whereas the expression levels of vos-1 were generally higher than those in the wild-type strain throughout the time course (Fig. 5A). These results suggest that FLB-3 is required for the proper expression of $a b a-1$, wet- 1 and vos-1 genes during vegetative growth.

The expression of the same genes was also analyzed during induction of asexual development (Fig. 5B). In the wild-type strain, $a b a-1$ expression decreased with time, while levels of the vos-1 transcript remained relatively constant during all time points analyzed. The maximum level of the wet- 1 transcript was observed at $9 \mathrm{~h}$ after induction of conidiation. In the $\Delta f l b-3$ strain however, very low levels of $a b a-1$ and wet- 1 transcripts were detected throughout the assay, indicating the requirement of FLB3 for the expression of both genes under this growth condition. On the other hand, levels of the vos-1 transcript were similar to those observed in the wild-type strain, suggesting that this gene may not be involved in conidiation in N. crassa as described in Aspergillus fumigatus (Tao and Yu, 2011) and A. nidulans (Ni and Yu, 2007). We suggest that FLB-3 directly regulates the expression of $a b a-1$, wet- 1 and vos- 1 genes, acting as an activator of $a b a-1$ and wet-1 expression under both vegetative growth and asexual development, and as a repressor of vos-1 expression under vegetative growth. Such regulation would be independent of the brlA regulator, whose ortholog was not identified in N. crassa. FLB-
3 appears not to be required for normal vos-1 expression under induction of conidiation.

In A. nidulans, using a GST pull-down assay, it was demonstrated that FlbC binds in vitro to the promoters of $\operatorname{brl} A, a b a A$ and $v o s A$, but not to that of wetA (Kwon et al., 2010). We identified putative FLB-3 binding sites in the $N$. crassa $a b a-1$, wet- 1 and vos- 1 promoters (Fig. 3B), and we investigated whether FLB-3 could bind in vivo to DNA fragments containing some of these motifs. Putative A. nidulans FlbC binding sites were also identified in the $N$. crassa wet- 1 and vos- 1 promoters (Fig. 3B), and one in the vos- 1 promoter was analyzed. For these experiments, we constructed a GFP-tagged FLB-3 overexpressing strain, by inserting the $f l b-3$ genomic sequence into the his-3 locus of a wild-type strain (his-3::Pccg-1$f l b-3-s f g f p$ ). The homokaryon strain (named as $f l b-3^{+}$) produces a C-terminal sfGFP-tagged FLB-3 under the control of the ccg-1 promoter. The FLB-3 overexpressing strain was compared to the wildtype strain under the same conditions, and we observed no differences in colony growth and in the amounts of macroconidia (Fig. S4A). Interestingly, the overexpressing strain grew more in 9d old cultures and produced a significantly higher number of microconidia than the wild-type strain $(\mathrm{p}<0.01$; approximately 400 times) (Fig. S4B), supporting a role for the FLB-3 transcription factor in $N$. crassa microconidiation.

ChIP-PCR assays were performed to analyze the binding of FLB-3-sfGFP to the aba-1, wet-1 and vos-1 promoters. Chromatin from mycelia after $12,24,48$, and $60 \mathrm{~h}$ of vegetative growth was used for immunoprecipitation, and binding of FLB-3 was analyzed by PCR using the oligonucleotides described in Table S1. The amplified DNA fragments (Fig. 6A) were quantified by ImageJ, and the results are shown in graphs (Fig. 6B). FLB-3 was able to bind to its predicted sites in the $a b a-1$ ( -2766 bp from the ATG start codon), vos-1 ( -3441 bp from the ATG start codon), and wet-1 ( $-2212 \mathrm{bp}$ from the ATG start codon) promoters after 48 and $60 \mathrm{~h}$ of growth $(p<0.01)$. However, we did not observe binding when mycelia collected at $12 \mathrm{~h}$ (data not shown) and $24 \mathrm{~h}$ (Fig. 6A) of vegetative growth were used. It is noteworthy that FLB-3 strongly bound to the $a b a-1$ and vos-1 promoters at both time points and binding to the wet-1 promoter appeared to be stronger at $48 \mathrm{~h}$ of growth (Fig. 6A and B). We also analyzed binding to a region of the vos-1 promoter that contains a match to the reported A. nidulans FlbC binding site (located at -759 bp from the ATG start codon). Interestingly, FLB-3 was unable to bind to the A. nidulans FlbC site located in vos-1 promoter, further supporting the FLB-3 DNA binding preferences determined by PBM. The results indicate that FLB-3 directly regulates the expression of these three genes, differently from what has been described in A. nidulans, where FlbC directly activates $b r l A$, the first gene of the conidiation cascade.

\subsection{Cross regulation among the aba-1, wet-1 and vos-1 genes}

The existence of genetic interactions between these genes was previously reported in A. fumigatus (Tao and Yu, 2011) and A. nidulans (Ni and Yu, 2007). In A. fumigatus, the AfuwetA transcript is not detected in the AfuabaA mutant, and AfuvosA expression is reduced in the AfuwetA and AfuabaA mutants, establishing an order of gene activation shown in Fig. 4A. In addition, in A. nidulans, wet $A$ levels strongly decrease in the $\triangle a b a A$ strain under vegetative growth condition ( $\mathrm{Ni}$ and $\mathrm{Yu}, 2007$ ). We thus decided to investigate the existence of genetic interactions among the $N$. crassa genes under vegetative growth and asexual development. We observed that $a b a-1$ and wet-1 down regulate each other under vegetative growth but they do not regulate vos-1 (Fig. 7A), although VOS-1 negatively regulates $a b a-1$ and wet- 1 (Fig. 7A). Under the same condition, FLB-3 directly regulates vos-1, as demonstrated in 
A
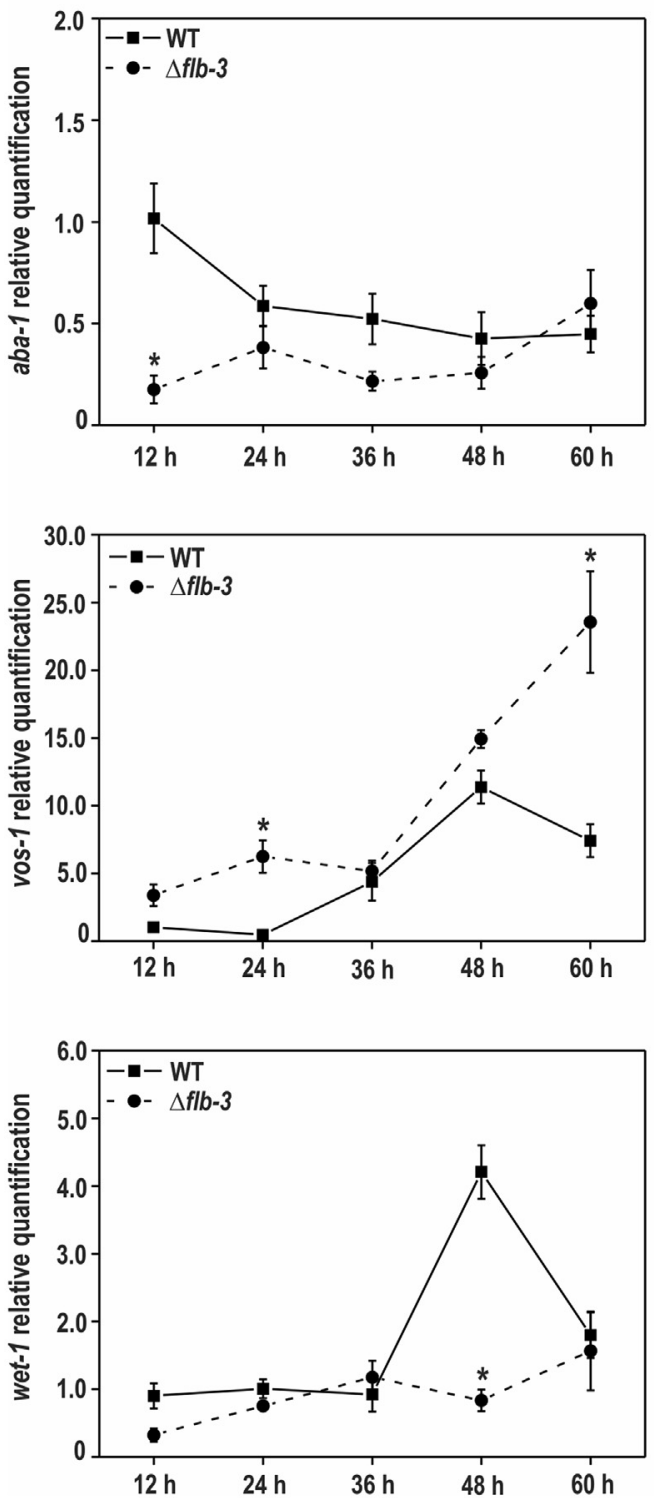

B
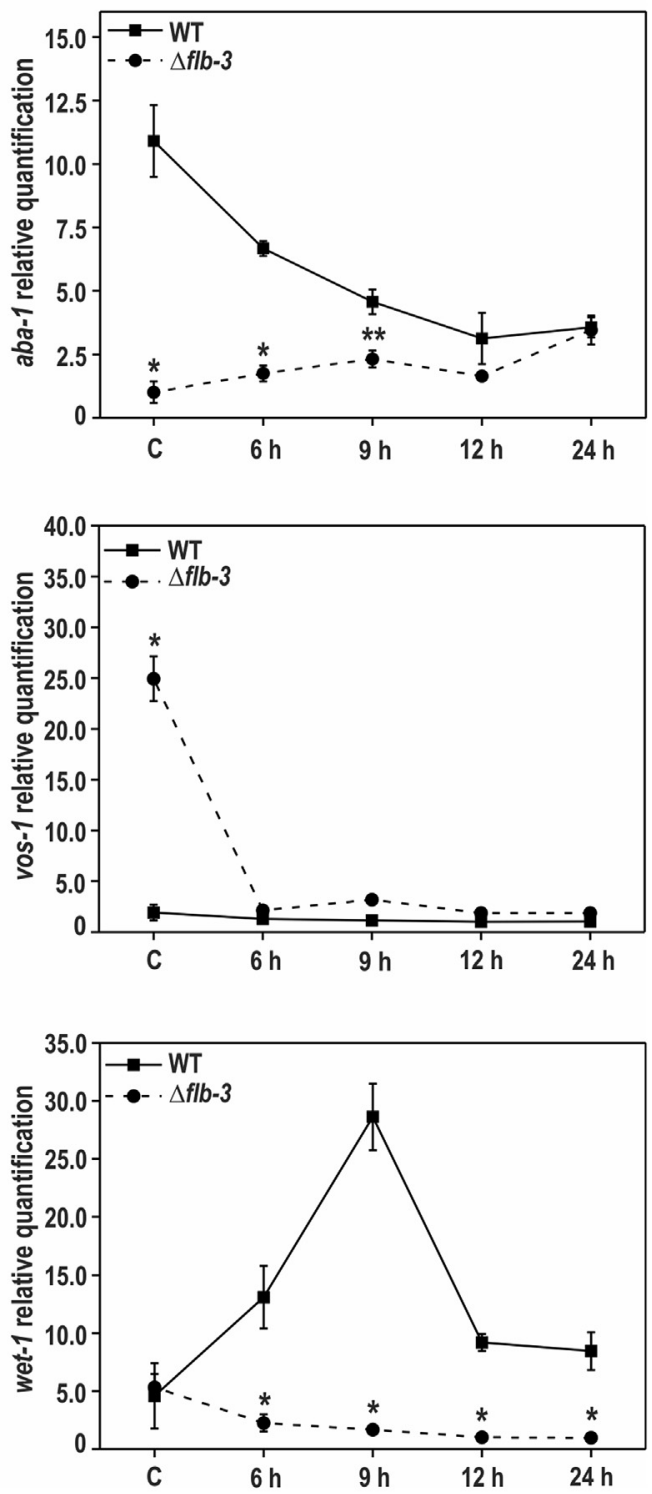

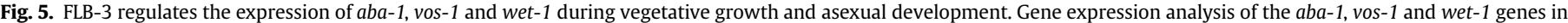

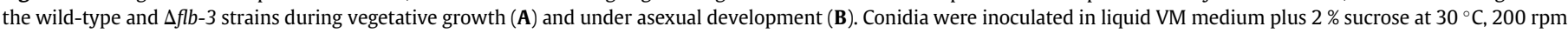

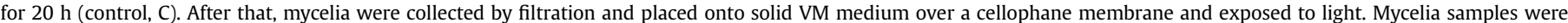

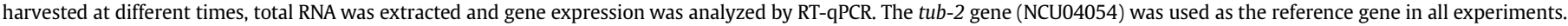

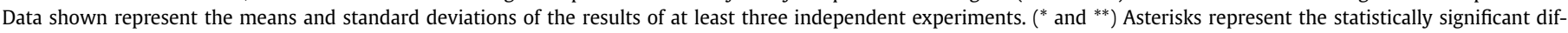

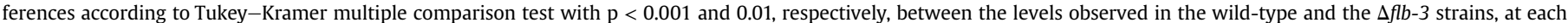
time point.

Fig. 5A. Under asexual development, the genetic interactions seem to be less prominent. Under this condition, ABA-1 does not regulate wet-1 and vos-1; however, both genes are required for proper regulation of $a b a-1$ expression, and a genetic interaction between wet- 1 and vos- 1 was only observed at $24 \mathrm{~h}$ of induction of conidiation (Fig. 7B). These results in $N$. crassa show differences in the genetic interaction when compared to Aspergilli, and indicate that the signaling pathway under control of FLB-3 is not fully conserved in distinctive filamentous fungi. Based on these results, we propose a model of interaction among $f l b-3, a b a-1$, wet- 1 and $v o s-1$ genes in $N$. crassa, which is based on a different order of gene activation under both growth conditions (Fig. 8).

\section{Discussion}

In this study, we investigated the functional role of the $N$. crassa FLB-3 transcription factor in developmental control and demonstrated that it has an important role in sexual and asexual development. In addition, we also characterized downstream genes, which are described as being under FLB-3 control in Aspergilli. This transcription factor has been characterized in only a few fungi, and in $N$. crassa it has only been previously identified in a screen for transcription factors regulating glycogen metabolism (Gonçalves et al., 2011), and as a gene regulated by light (Wu et al., 2014) and during development (Greenwald et al., 2010). Deletion of $f l b-3$ 
A

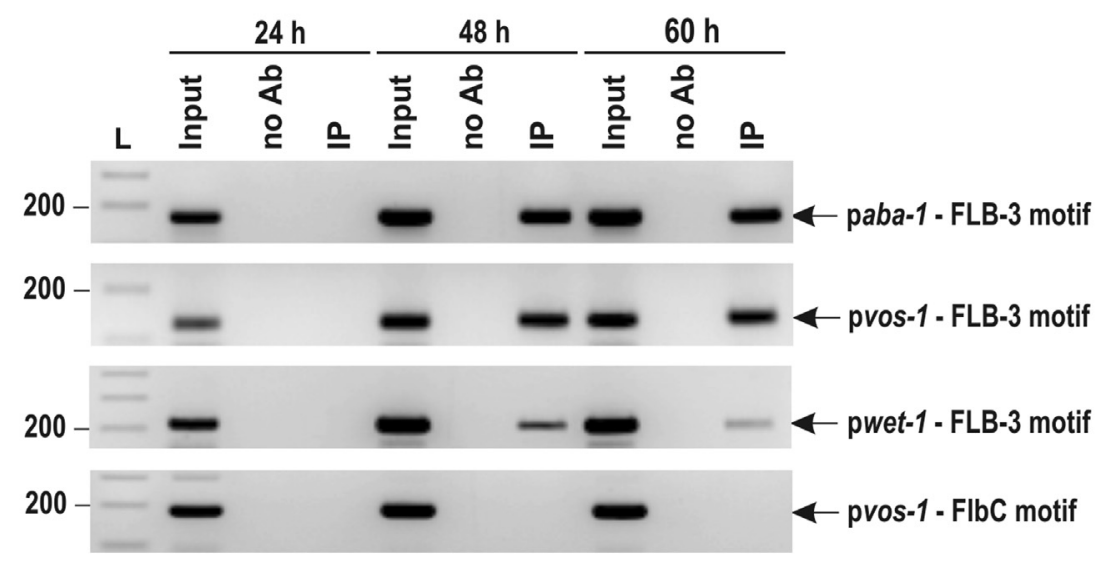

B
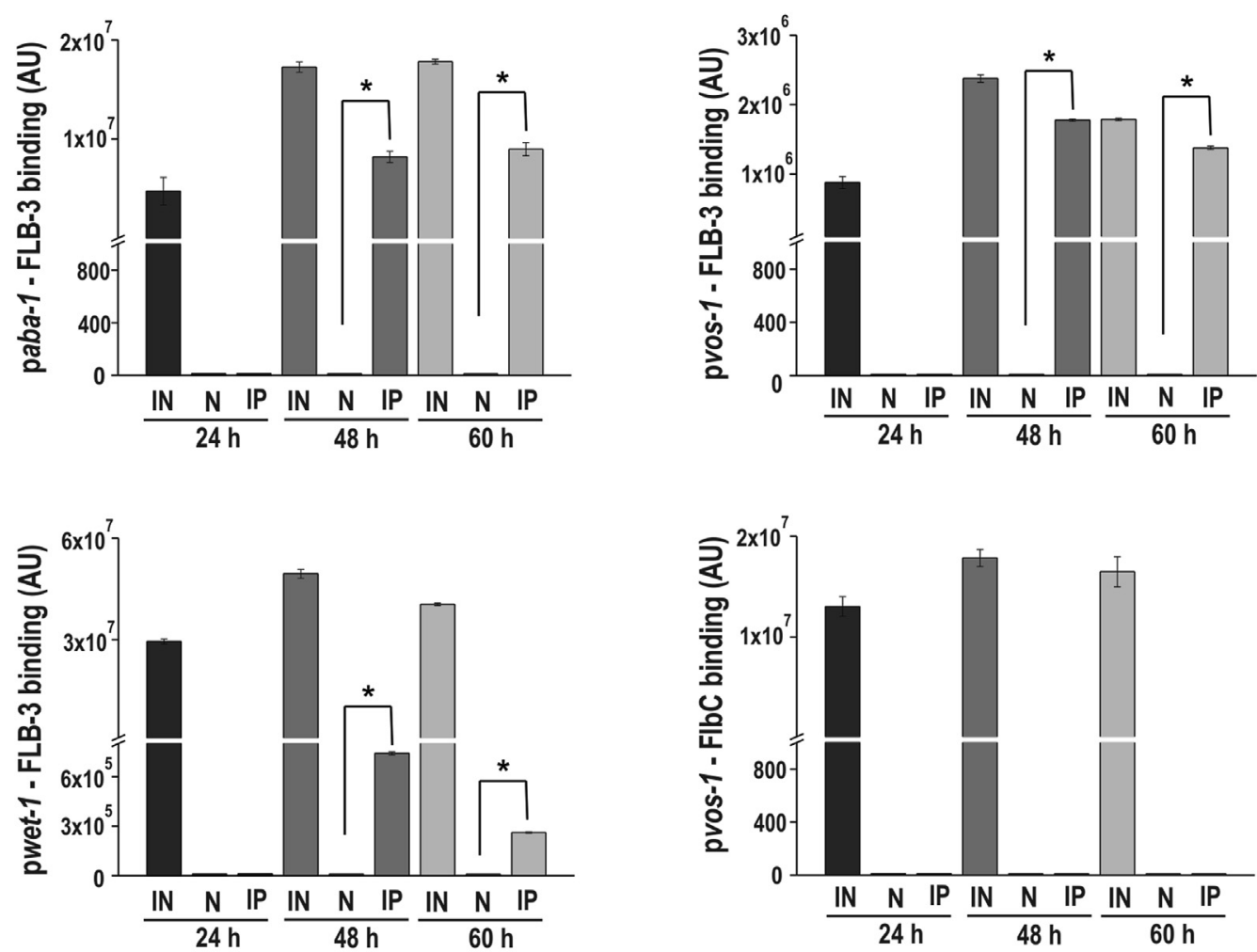

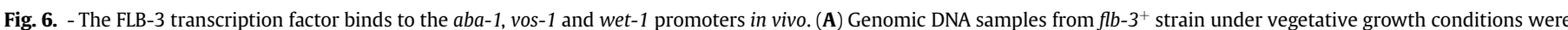

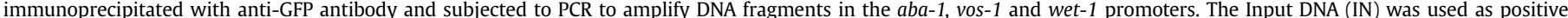

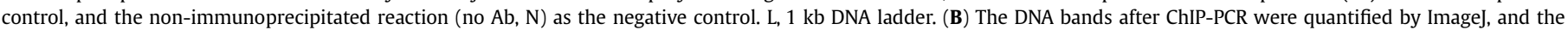

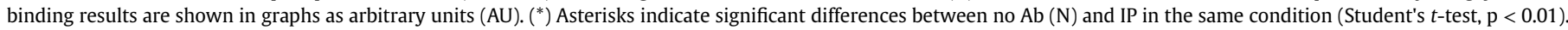
The results represent the average of two independent experiments.

results in severe defects in conidiation and in sterility. While the production of the female sexual structure was increased, similar to the phenotype described for the A. nidulans $\Delta f l b C$ strain (Kwon et al., 2010), the protoperithecia were unable to cross.

In $A$. nidulans, FlbC is necessary for proper activation of conidiation, acting as an activator of $\operatorname{brl} A$, the first gene of the $b r l A \rightarrow a b a A \rightarrow$ wetA cascade that defines the central regulatory pathway, and controls conidiation in this organism (Kwon et al., 2010; Park and Yu, 2012; Mirabito et al., 1989). The A. nidulans regulators VosA and VelB have been shown to be interconnected to this regulatory pathway via negative feedback regulation of $\mathrm{brlA}$ and other developmental genes (Park and Yu, 2012; Ni and Yu,
2007). In addition, they function in spore maturation, by controlling trehalose biogenesis and wall completion (Park and Yu, 2012; Ni and Yu, 2007). In P. anserina, a fungus more closely related to N. crassa, FLE-1 (the FLB-3 ortholog) coordinates male and female sexual differentiation, by controlling the balance between male and female structure production (Coppin, 2002), and in F. verticillioides, this protein, termed Ada1, plays a role in asexual development (Malapi-Wight et al., 2014). Therefore, this transcription factor appears to be involved in the control of the transition from vegetative growth to asexual/sexual development in fungi, but the precise mechanisms through which it performs this function depend on the organism. While in $A$. nidulans $f l b C$ acts upstream of 
A
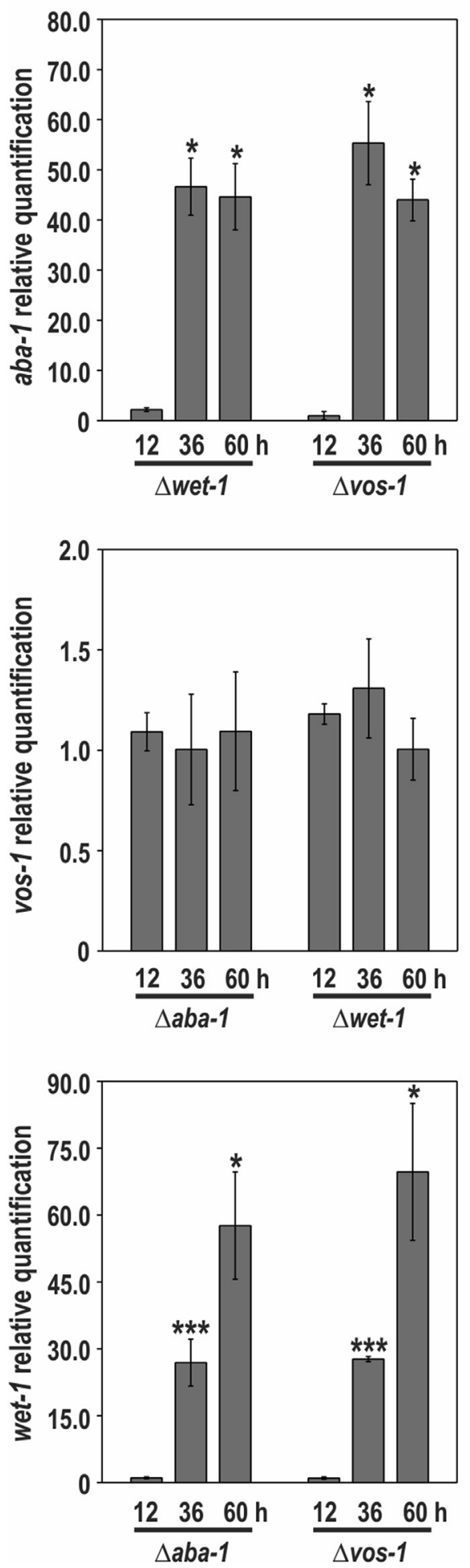

B
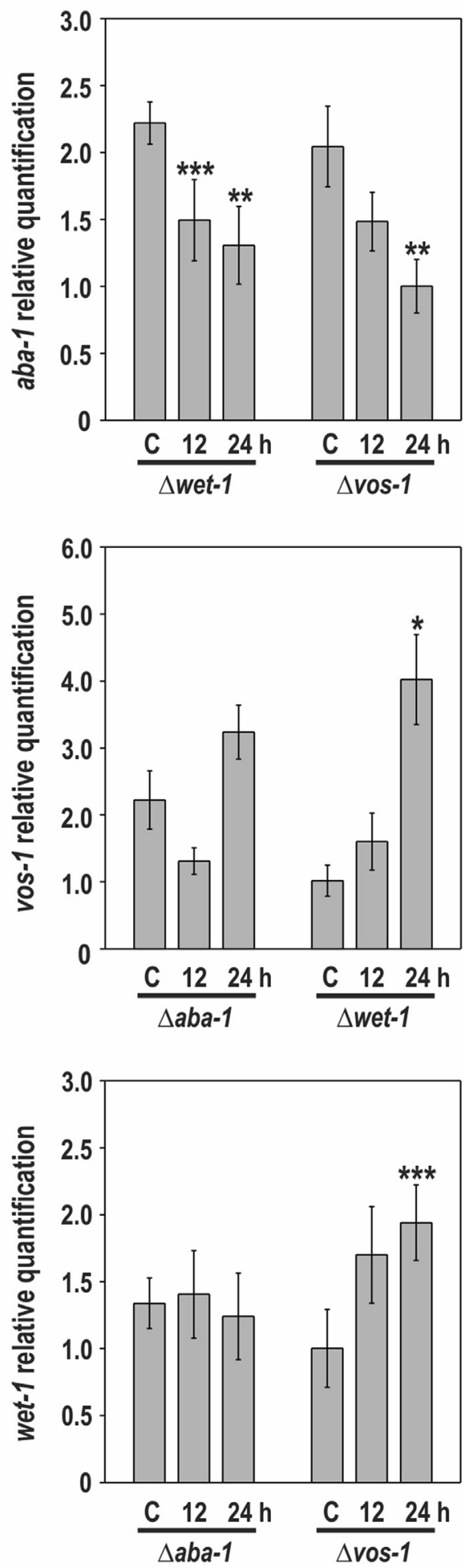

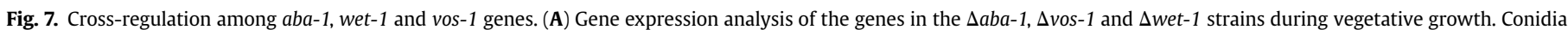

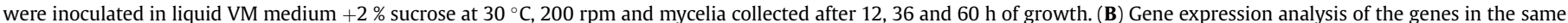

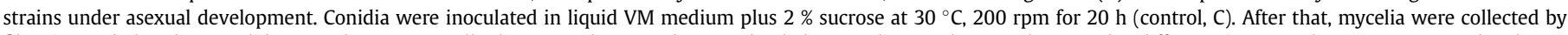

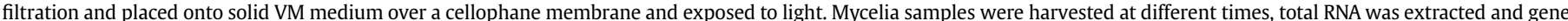

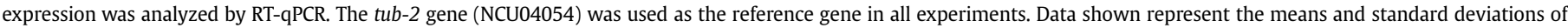

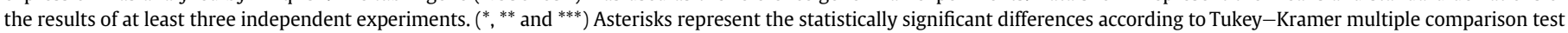
with $\mathrm{p}<0.001,0.01$ and 0.05 , respectively, between the levels observed each time point relative to time $12 \mathrm{~h}(\mathbf{A})$ and to C (B). 

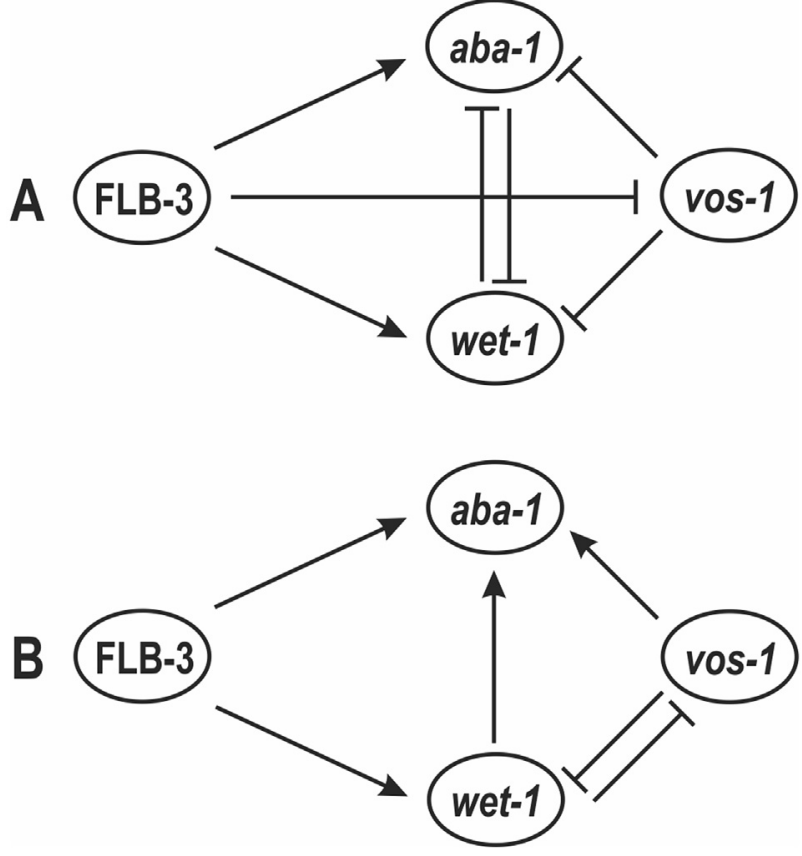

Fig. 8. Simplified models of the role of FLB-3 in the regulation of $a b a-1$, wet-1 and vos1 expression during vegetative growth (A) and under induction of asexual development (B) in $N$. crassa. The genetic interactions among the aba-1, wet-1 and vos-1 genes are shown. The regulatory mechanisms and the genetic interactions among the genes are presented in the text.

brlA, regulating the expression of conidiation-specific genes, in $P$. anserina, $F$. verticillioides and in $N$. crassa (as shown in this study), its role in conidiation appears to be independent of the $\mathrm{brlA}$ gene, since this protein was not identified in any of these organisms (Coppin, 2002; Malapi-Wight et al., 2014). Although FlbC has been reported to play a direct role in the expression of the $a b a A$ and vos $A$ genes, independent of $\operatorname{brl} A$, activation of $\operatorname{brl} A$ is required for conidiophore development in A. nidulans (Kwon et al., 2010).

The DNA-binding specificity of FLB-3 was determined here by protein binding microarrays and we demonstrated that this transcription factor binds in vivo to the predicted cis elements present in the $a b a-1$, wet- 1 and vos-1 promoters. However, under the same conditions, it does not bind to a predicted FlbC binding site that exists in the vos-1 promoter. The FLB-3 motif we determined here was expected to be similar to that determined for FlbC, the A. nidulans ortholog of FLB-3 (Kwon et al., 2010), considering the high sequence identity exhibited by these proteins in their DNAbinding regions (Weirauch et al., 2014; Bernard et al., 2012). The motifs, however, are only somewhat similar, and we argue that the motif reported herein is more likely to represent the true DNAbinding specificity of FlbC orthologs. The main reasons are that the reported FlbC motif was based only on a handful of promoters, and that our motif is not only in large agreement with that determined for a related protein (YPR013C) (Zhu et al., 2009), but is also consistent with that derived from predictive models for DNAbinding specificity of zinc finger proteins (Gupta et al., 2014; Najafabadi et al., 2015). The previously reported motif for FlbC, on the other hand, performs poorly in both analyses. Interestingly, an alternative motif for FlbC, GTAGATC, has previously been presented that better resembles the one of YPR013C, and consequently, also of FLB-3 (Kwon et al., 2010). Based on our results, we consider it to be a more accurate representation of its DNA-binding preference. Altogether, our analyses suggest that the FLB-3 motif we report here better represents the DNA-binding preference of FlbC orthologs, including that of $A$. nidulans FlbC.
The $N$. crassa aba-1, wet- 1 and vos- 1 genes were identified in this work, and we showed that these genes may not play a role in the induction of conidiation in $N$. crassa, differing from what has been described for their orthologs in A. nidulans (Adrianopoulos and Timberlake, 1994), A. fumigatus (Tao and Yu, 2011) and Fusarium graminearum (Son et al., 2013). Loss-of-function mutation in $a b a A$ of $A$. nidulans and A. fumigatus has been shown to result in aberrant conidiophores exhibiting cylinder-like terminal cells without conidia (Adrianopoulos and Timberlake, 1994; Tao and Yu, 2011). Deletion of $\operatorname{vos} A$ in $A$. nidulans leads to a drastic reduction in conidia viability and apparent lack of cytoplasm and organelle (Ni and Yu, 2007). In addition, deletion of the A. fumigatus wetA gene ( $A f u-$ wetA) affects conidia wall formation, resulting in incomplete conidial separation and maturation, and reduced viability when compared to the wild-type strain (Tao and Yu, 2011). In F. graminearum, deletion of wetA results in longer conidia, containing fewer septa than those of the wild-type strain. Additionally, the conidia are sensitive to oxidative and heat stresses (Son et al., 2014). The corresponding $N$. crassa knockout strains, on the other hand, conidiate well and their conidia show high viability over time. These genes may play additional roles in $N$. crassa, similar to what has been described for $a b a A$ in A. nidulans, where deletion of $a b a A$ affects autolysis by reducing chitinase production (Emri et al., 2008). Although seemingly not involved in conidiation in N. crassa, these genes do appear to rely on FLB-3 for proper expression, which we report here as a regulator of conidiation, during both vegetative growth and in the induction of asexual development. Alternatively, these proteins may indeed play a role in conidiation, but additional proteins may compensate for their function in their absence. It is important to emphasize that although FLB-3 regulates the expression of $a b a-1$, wet- 1 and vos-1 genes in the absence of brlA, its role in the regulation of conidiation is, most likely, independent of these genes considering the phenotypes of the three mutants.

We demonstrated here that FLB-3 is involved in sexual and asexual development in $N$. crassa, and the gene overexpression data suggests that it specifically impacts the microconidiation process. Moreover, FLB-3 binds to the promoter of the aba-1, wet-1 and vos-1 genes and their expression is misregulated in the $\Delta f b-3$ strain, suggesting that they are direct targets of this transcription factor and their function might be connected to FLB-3. However, mutant strains in these three genes conidiate well. Our data also show that regulatory interactions among the aba-1, wet- 1 and vos- 1 genes are less prominent under asexual development than those observed during vegetative growth, which reinforces that these genes may not play an important role in conidiation. Therefore, the role of FLB3 in conidiation is independent of these three genes. Their promoters can be bound in vivo by FLB-3, which raises questions about the functions of these genes in $N$. crassa. The velvet regulators, which include the VOS-1 protein, are involved in important biological process including tolerance to stress (Park et al., 2012; Lan et al., 2014). However, in N. crassa, a systematic analysis of the $\Delta v o s-1$ strain under different stress conditions revealed that VOS-1 may not play a role in stress response (unpublished results). Considering that the role of these genes may differ from what has been described in other filamentous fungi, investigating their targets may be useful in fully understanding these proteins in $N$. crassa. Although much work remains to be done, this report represents a first step in understanding the functional role of the FLB-3 transcription factor in N. crassa, which can be further used as an entry point for the study of how regulatory circuits involved in development change among different fungal species.

\section{Conflicts of interest}

The authors declare no conflict of interest. 


\section{Acknowledgments}

We thank the Fungal Genetics Stock Center (Manhattan, KS, USA) for the N. crassa strains. We would like to thank the Fundação de Amparo à Pesquisa do Estado de São Paulo (FAPESP, Proc. 2013/ 24705-3) and Conselho Nacional de Desenvolvimento Científico e Tecnológico (CNPq) (Grant no. 444068/2014-7) for providing research grants to MCB and fellowships to DLA, ACB, FBC, SV, FZF, FAC, RDG, and MCB. LFL and AMM are supported by grants from Millennium Institute for Integrative Systems and Synthetic Biology (MIISSB) and Fondo Nacional de Desarrollo Científico y Tecnológico (FONDECYT 1171151). TRH acknowledges support from the Canadian Institutes of Health Research (FDN-148403). We also acknowledge the support for the International Symposium on Fungal Stress (ISFUS)-2017 meeting from the Coordenação de Aperfeiçoamento de Pessoal de Nível Superior (CAPES) of Brazil, PAEP 88887.126652/2017-00 and the Fundação de Amparo à Pesquisa do Estado de Goiás of Brazil, 201710267000110.

\section{Appendix A. Supplementary data}

Supplementary data related to this article can be found at https://doi.org/10.1016/j.funbio.2018.01.004.

\section{References}

Adams, T.H., Boylan, M.T., Timberlake, W.E., 1988. brlA is necessary and sufficient to direct conidiophores development in Aspergillus nidulans. Cell 54, 353-362.

Adams, T.H., Wieser, J.K., Yu, J.-H., 1998. Asexual sporulation in Aspergillus nidulans. Microbiol. Mol. Biol. Rev. 62, 35-54.

Adrianopoulos, A., Timberlake, W.E., 1991. ATTS, a new and conserved DNA binding domain. Plant Cell 3, 747-748.

Adrianopoulos, A., Timberlake, W.E., 1994. The Aspergillus nidulans abaA gene encodes a transcriptional activator that acts as a genetic switch to contro development. Mol. Cell Biol. 14, 2503-2515.

Andrilenas, K.K., Penvose, A., Siggers, T., 2015. Using protein-binding microarrays to study transcription factor specificity: homologs, isoforms and complexes. Brief. Funct. Genom. 14, 17-29.

Bayram, O., Braus, G.H., 2012. Coordination of secondary metabolism and development in fungi: the velvet family of regulatory proteins. FEMS Microbiol. Rev. $36,1-24$.

Berger, M.F., Philippakis, A.A., Qureshi, A.M., He, F.S., Estep 3rd, P.W., Bulyk, M.L., 2006. Compact, universal DNA microarrays to comprehensively determine transcription-factor binding site specificities. Nat. Biotechnol. 24, 1429-1435.

Bernard, B., Thorsson, V., Rovira, H., Shmulevich, I., 2012. Increasing coverage of transcription factor position weight matrices through domain-level homology. PLoS One 7, e42779.

Campbell, S., Inamdar, M., Rodrigues, V., Raghavan, V., Palazzolo, M., Chovnick, A 1992. The scalloped gene encodes a novel, evolutionarily conserved transcription factor required for sensory organ differentiation in Drosophila. Genes Dev 3, 367-379.

Chung, W., 2011. Assessing Conserved Function of Conidiation Regulators in Two Distantly Related Ascomycetes, Aspergillus nidulans and Neurospora crassa. Dissertation. http://oaktrust.library.tamu.edu/bitstream/handle/1969.1/ETDTAMU-2011-05-9321/CHUNG-DISSERTATION.pdf? sequence $=2$

Coppin, E., 2002. The fle1 gene encoding a $\mathrm{C}_{2} \mathrm{H}_{2}$ zinc finger protein co-ordinates male and female sexual differentiation in Podospora anserina. Mol. Microbiol. 43, 1255-1268.

Ebbole, D., Sachs, M.S., 1990. A rapid and simple method for isolation of Neurospora crassa homokaryons using microconidia. Fungal Genet. Rep. 37.

Eddy, S.R., 2009. A new generation of homology search tools based on probabilistic inference. Genome Inform. 23, 205-211.

Emri, T., Molnár, Z., Szilágyi, M., Pócsi, I., 2008. Regulation of autolysis in Aspergillus nidulans. Appl. Biochem. Biotechnol. 151, 211-220.

Freitas, F.Z., Virgilio, S., Cupertino, F.B., Kowbel, D.J., Fioramonte, M., Gozzo, E.C Glass, N.L., Bertolini, M.C., 2016. The SEB-1 transcription factor binds to the STRE motif in Neurospora crassa and regulates a variety of cellular processes including the stress response and reserve carbohydrate metabolism. G3 Genes Genom. Genet. 6, 1327-1343.

Gonçalves, R.D., Cupertino, F.B., Freitas, F.Z., Luchessi, A.D., Bertolini, M.C., 2011 A genome-wide screen for Neurospora crassa transcription factors regulating glycogen metabolism. Mol. Cell. Proteomics 10. https://doi.org/10.1074/ mcp.M111.007963-2.
Greenwald, C.J., Kasuga, T., Glass, N.L., Shaw, B.D., Ebbole, D.J., Wilkinson, H.H., 2010. Temporal and spatial regulation of gene expression during asexual development of Neurospora crassa. Genetics 186, 1217-1230.

Gupta, S., Stamatoyannopoulos, J.A., Bailey, T.L., Noble, W.S., 2007. Quantifying similarity between motifs. Genome Biol. 8, R24.

Gupta, A., Christensen, R.G., Bell, H.A., Goodwin, M., Patel, R.Y., Pandey, M. Enuameh, M.S., Rayla, A.L., Zhu, C., Thibodeau-Beganny, S., Brodsky, M.H., Joung, J.K., Wolfe, S.A., Stormo, G.D., 2014. An improved predictive recognition model for Cys2-His2 zinc finger proteins. Nucleic Acids Res, 42, 4800-4812.

Kays, A.M., Borkovich, K.A., 2004. Severe impairment of growth and differentiation in a Neurospora crassa mutant lacking all heterotrimeric $\mathrm{G}$ alpha proteins. Genetics $166,1229-1240$.

Kwon, N.-J., Garzia, A., Espeso, E.A., Ugalde, U., Yu, J.-H., 2010. FlbC is a putative nuclear $\mathrm{C}_{2} \mathrm{H}_{2}$ transcription factor regulating development in Aspergillus nidulans. Mol. Microbiol. 77, 1203-1219.

Laloux, I., Dubois, E., Dewerchin, M., Jacobs, E., 1990. TEC-1, a gene involved in the activation of Tyl and Tyl-mediated gene expression in Saccharomyces cerevisiae: cloning and molecular analysis. Mol. Cell Biol. 10, 3541-3550.

Lan, N., Zhang, H., Hu, C., Wang, W., Calvo, A.M., Harris, S.D., Chen, S., Li, S., 2014. Coordinated and distinct functions of velvet proteins in Fusarium verticillioides. Eukaryot. Cell 13, 909-918.

Livak, K.J., Schmittgen, T.D., 2001. Analysis of relative gene expression data using real-time quantitative PCR and the 2(-Delta Delta C(T)) method. Methods 25, 402-408.

Malapi-Wight, M., Kim, J.-E., Shim, W.-B., 2014. The N-terminus region of the putative $\mathrm{C}_{2} \mathrm{H}_{2}$ transcription factor Ada1 harbors a specie-specific activation motif that regulates asexual reproduction in Fusarium verticillioides. Fungal Genet. Biol. 62, 25-33.

Marshall, M.A., Timberlake, W.E., 1991. Aspergillus nidulans wetA activates sporespecific gene expression. Mol. Cell Biol. 11, 55-62.

McCluskey, K., 2003. The fungal genetics stock center: from molds to molecules. Adv. Appl. Microbiol. 52, 245-262.

Mirabito, P.M., Adams, T.H., Timberlake, W.E., 1989. Interactions of three sequentially expressed genes control temporal and spatial specificity in Aspergillus development. Cell 57, 859-868.

Najafabadi, H.S., Mnaimneh, S., Schmitges, F.W., Garton, M., Lam, K.N., Yang, A., Albu, M., Weirauch, M.T., Radovani, E., Kim, P.M., Greenblatt, J., Frey, B.J., Hughes, T.R., 2015. $\mathrm{C}_{2} \mathrm{H}_{2}$ zinc finger proteins greatly expand the human regulatory lexicon. Nat. Biotechnol. 33, 555-562.

Ni, M., Yu, J.-H., 2007. A novel regulator couples sporogenesis and trehalose biogenesis in Aspergillus nidulans. PLoS One 10, e970.

Park, H.-S., Yu, J.-H., 2012. Genetic control of asexual sporulation in filamentous fungi. Curr. Opin. Microbiol. 15, 669-677.

Park, H.-S., Bayram, O., Braus, G.H., Kim, S.C., Yu, J.-H., 2012. Characterization of the velvet regulators in Aspergillus fumigatus. Mol. Microbiol. 86, 937-953.

Sokolovsky, V., Kaldenhoff, R., Ricci, M., Russo, V.E.A., 1990. Fast and reliable miniprep RNA extraction from Neurospora crassa. Fungal Genet. Newslett. 37.

Son, H., Kim, M.G., Min, K., Seo, Y.S., Lim, J.Y., Choi, G.J., Kim, J.C., Chae, S.K., Lee, Y.W., 2013. AbaA regulates conidiogenesis in the ascomycete fungus Fusarium graminearum. PLoS One 8, e72915.

Son, H., Kim, M.G., Min, K., Lim, J.Y., Choi, G.J., Kim, J.C., Chae, S.K., Lee, Y.W., 2014 WetA is required for conidiogenesis and conidium maturation in the ascomycete fungus Fusarium graminearum. Eukaryot. Cell 13, 87-98.

Springer, M.L., Yanofsky, C., 1989. A morphological and genetic analysis of conidiophores development in Neurospora crassa. Genes Dev. 3, 559-571.

Stormo, G.D., 1990. Consensus patterns in DNA. Methods Enzymol. 183, 211-221.

Tamaru, H., Zhang, X., McMillen, D., Singh, P.B., Nakayama, J., Grewal, S.I., Allis, C.D., Cheng, X., Selker, E.U., 2003. Trimethylated lysine 9 of histone H3 is a mark for DNA methylation in Neurospora crassa. Nat. Genet. 34, 75-79.

Tao, L., Yu, J.-H., 2011. AbaA and WetA govern distinct stages of Aspergillus fumigatus development. Microbiology 157, 313-326.

Vogel, H.J., 1956. A convenient growth medium for Neurospora crassa (medium N). Microb. Genet. Bull. 13, 42-43.

Wasserman, W.W., Sandelin, A., 2004. Applied bioinformatics for the identification of regulatory elements. Nat. Rev. Genet. 5, 276-287.

Weirauch, M.T., Cote, A., Norel, R., Annala, M., Zhao, Y., Riley, T.R., Saez-Rodriguez, J. Cokelaer, T., Vedenko, A., Talukder, S., , DREAM5 Consortium, Bussemaker, H.J., Morris, Q.D., Bulyk, M.L., Stolovitzky, G., Hughes, T.R., 2013. Evaluation of methods for modeling transcription factor sequence specificity. Nat. Biotechnol. 31, 126-134.

Weirauch, M.T., Yang, A., Albu, M., Cote, A.G., Montenegro-Montero, A., Drewe, P., Najafabadi, H.S., Lambert, S.A., Mann, I., Cook, K., Zheng, H., Goity, A., van Bakel, H., Lozano, J.C., Galli, M., Lewsey, M.G., Huang, E., Mukherjee, T., Chen, X., Reece-Hoyes, J.S., Govindarajan, S., Shaulsky, G., Walhout, A.J.M., Bouget, F.Y., Ratsch, G., Larrondo, L.F., Ecker, J.R., Hughes, T.R., 2014. Determination and inference of eukaryotic transcription factor sequence specificity. Cell 158, $1431-1443$.

Westergaard, M., Mitchell, H.K., 1947. Neurospora V. A synthetic medium favoring sexual reproduction. Am. J. Bot. 34, 573-577.

Wieser, J., Lee, B.N., Fondon 3rd, J.W., Adams, T.H., 1994. Genetic requirements for initiating asexual development in Aspergillus nidulans. Curr. Genet. 27, 62-69.

Wu, C., Yang, F., Smith, K.M., Peterson, M., Dekhang, R., Zhang, Y., Zucker, J. Bredeweg, E.L., Mallappa, C., Zhou, X., Lyubetskaya, A., Townsend, J.P., 
Galagan, J.E., Freitag, M., Dunlap, J.C., Bell-Pedersen, D., Sachs, M.S., 2014. Genome-wide characterization of light-regulated genes in Neurospora crassa. G3 Genes Genom. Genet. 4, 1731-1745.

Xiao, J.H., Davidson, I., Matthes, H., Garnier, J.M., Chambon, P., 1991. Cloning, expression and transcription properties of the human enhancer factor TEF-1. Cell 17, 551-568.

Yao, G., Li, Z., Wu, R., Qin, Y., Liu, G., Qu, Y., 2016. Penicillium oxalicum PoFlbC regulates fungal asexual development and is important for cellulase gene expression. Fungal Genet. Biol. 86, 91-102.
Zhao, Y., Stormo, G.D., 2011. Quantitative analysis demonstrates most transcription factors require only simple models of specificity. Nat. Biotechnol. 29, 480-483.

Zhu, C., Byers, K.J., McCord, R.P., Shi, Z., Berger, M.F., Newburger, D.E., Saulrieta, K., Smith, Z., Shah, M.V., Radhakrishnan, M., Philippakis, A.A., Hu, Y., De Masi, F., Pacek, M., Rolfs, A., Murthy, T., Labaer, J., Bulyk, M.L., 2009. High-resolution DNA-binding specificity analysis of yeast transcription factors. Genome Res. 19, 556-566. 